\title{
A Taxonomic Study on the Burrowing Cricket Genus Velarifictorus with Morphologically Resembled Genus Lepidogryllus (Orthoptera: Gryllidae: Gryllinae) in Korea
}

\author{
Tae-Woo Kim* \\ National Institute of Biological Resources, Incheon 404-170, Korea
}

\begin{abstract}
The burrowing-cricket genus Velarifictorus Randell, 1964 is reviewed in Korea, comparing with morphologically resembled genus Lepidogryllus Otte and Alexander, 1983 for the first time. First, Velarifictorus aspersus borealis Gorochov, 1985 is confirmed from only restricted area of southern regions in Gyeongsangnam-do and Jeollanam-do. Second, Velarifictorus micado (Saussure, 1877) is confirmed from nearly all around the Korean peninsula including North Korea. Third, the previously not recorded Velarifictorus ornatus (Shiraki, 1911) is newly recognized from South Korea. Relating to the genus Velarifictorus, the resembled genus Lepidogryllus Otte and Alexander, 1983 and its species Lepidogryllus siamensis (Chopard, 1961) com. \& stat. nov. is studied and compared with Velarifictorus members. A key, descriptions, tables, photographs, figures, oscillograms and spectrograms of calling sounds are provided to aid identification between the four similar species.
\end{abstract}

Keywords: taxonomy, Orthoptera, Gryllidae, Gryllinae, Velarifictorus, Lepidogryllus, new record, Korea

\section{INTRODUCTION}

The burrowing cricket genus Velarifictorus Randell, 1964 was distinguished from the previously known genus Scapsipedus Saussure, 1877. Both genera are rather similar with each other in external characters such as the male's enlarged round head with swollen frons, but there were main differences in the genitalic structures of males (Randell, 1964). Each of them belong to different tribes, those are Velarifictorus in the Modicogryllini, against Scapsipedus in the Gryllini (Otte, 1994). Three subgenera (Velarifictorus Randell, 1964; Buangina Otte and Alexander, 1983; Pseudocoiblemmus Gorochov, 2001) with a hundred species are distributed throughout tropical Asia, Australia, and Africa (Eades et al., 2013). One of the members, the Japanese burrowing cricket Velarifictorus micado, was introduced and successfully established into North America (Alexander and Walker, 1962; Walker, 1977).

In East Asia, around the Korean peninsula, the numbers of Velarifictorus are estimated in the neighboring countries as follows: nine in China (Hua, 2000), five in Japan (Ichikawa et al., 2000), and only one in Far Eastern Russia (Storozhenko, 2004). In Korea, three of the Velarifictorus had been cited, those were Velarifictorus aspersus, Velarifictorus micado and Velarifictorus parvus by Kwon and Huh (1994) and Kwon et al. (1996). Velarifictorus aspersus is a widespread cricket in South Asian countries, having remarkable morphological variations (Ingrisch, 1998). Since the first record of Velarifictorus aspersus (="Gryllodes berthellus") from Seoul (="Keijo") by Shiraki (1930), there are a lot of records that had been referred in Korea, including junior synonyms. Therefore, it has been regarded as the commonest cricket in Korea. The other Velarifictorus micado was lately listed by Kwon and Huh (1994), but without any remarks or presentation of specimen data. Velarifictorus parvus was last recorded by Choo and Choi (1983), however, its identification was corrected to the other genus and species Modicogryllus siamensis by Paik et al. (2010), but they noted that its distribution is quite questioned in Korea.

In the course of taxonomic studies on Korean crickets and (c) This is an Open Access article distributed under the terms of the Creative Commons Attribution Non-Commercial License (http://creativecommons.org/ licenses/by-nc/3.0/) which permits unrestricted non-commercial use, distribution, and reproduction in any medium, provided the original work is properly cited.

pISSN 2234-6953 eISSN 2234-8190
*To whom correspondence should be addressed

Tel: 82-32-590-7433, Fax: 82-32-590-7436

E-mail: pulmuchi@korea.kr 
its sound recording projects, the following results were produced relating to the genus Velarifictorus. At first, most early recorded Velarifictorus aspersus are reconfirmed, but the result in this study shows that its distributional ranges are indeed restricted to only several far southern localities in Gyeongsangnam-do and Jeollanam-do. Comparing specimens with prior descriptions, Korean populations should be considered as northern subspecies Velarifictorus aspersus borealis, as pointed out by Storozhenko and Paik (2007). Second, the lately listed Velarifictorus micado is confirmed from nearly all around the Korean peninsula including North Korea (Kim and Puskás, 2012). Judging from these distributional data, it shows that Velarifictorus micado is the most common cricket in Korea rather than Velarifictorus aspersus borealis. Previous records of Velarifictorus aspersus might be misidentified Velarifictorus micado. Third, the previously not reported Velarifictorus sp. was newly recognized, and its sounds were successfully recorded in South Korea (audio available in Kim, 2011). Herein it is identified as Velarifictorus ornatus (Shiraki, 1911), the cricket recorded from Taiwan (type-locality), China (Yin and Liu, 1995), and Japan (Ichikawa et al., 2000).

On the other hand, Modicogryllus siamensis was also confirmed, and its sound data was presented in South Korea (Kim, 2011). The cricket is confused with Velarifictorus members because of the similarity of external characters, but distinguishing characters were well given by Ingrisch (1998). As regard to the genus Modicogryllus, four subgenera (Modicogryllus Chopard, 1961; Eumodicogryllus Gorochov, 1986; Promodicogryllus Gorochov, 1986; Amodicogryllus Gorochov, 1996) with 88 members are widely distributed in Asia, Europe and Africa (Eades et al., 2013). It has been partly underway to review this, such as the following steps: first species grouping (Gorochov, 1978), new subgeneric establishment (Gorochov, 1986), and finally distinct generic consideration (Gorochov, 1993). But a lot of members are still combined in a broad sense with Modicogryllus, because of previous studies treated only a part of the Middle Asian species. A comprehensive revision on the Modicogryllus with the allied subgenera is needed (Ingrisch, 1998).

In the case of Modicogryllus siamensis, for which a subgeneric position was not even assigned (Ingrisch, 1998), it was successively posited as Modicogryllus (Lepidogryllus) siamensis by Ichikawa et al. (2000). The primarily independent genus Lepidogryllus Otte and Alexander, 1983 was established on the basis of Australian two crickets, the other one species was described from Vietnam (Gorochov, 1991). Although there is no congruence regarding its position being either set under Modicogryllus, or being a separate genus, Lepidogryllus can be characterized by the male's enlarged spermatophore sac, compared with typical Modicogryllus
(Gorochov, 1991; Ichikawa et al., 2000). These genitalic structures are used as the principal diagnostic characters to consider generic levels (Chopard, 1961; Randell, 1964). In order to preserve the original authority of the genus, herein Lepidogryllus siamensis com. \& stat. nov. is regarded apart from Modicogryllus.

The aim of this paper is to review the burrowing cricket genus Velarifictorus with the morphologically resembled genus Lepidogryllus from Korea based on the result of the project concerned with morphological and acoustical studies on Korean crickets. The examined materials are about 300 specimens, mostly deposited in the collections of the National Institute of Biological Resources (Incheon). A few specimens were additionally studied in the National Academy of Agricultural Science (Suwon), Gyeongsang National University (Jinju), and Ewha Women's University (Seoul). The terminology for genitalic description refers to Randell (1964), and Gorochov (1978). The male's calling sounds were recorded by digital recorder (PCM-D50; Sony, Tokyo, Japan) with sampling mode $44-96 \mathrm{kHz}, 24$ bit in an acoustic room. Audio digital files were analyzed by Raven Pro 1.4 (Cornell Lab of Ornithology), to make out its oscillograms and spectrograms.

\section{SYSTEMATIC ACCOUNTS}

Order Orthoptera Olivier, 1789

Family Gryllidae Laicharting, 1781

Subfamily Gryllinae Laicharting, 1781

\section{Genus Velarifictorus Randell, 1964}

Type species: Scapsipedus micado Saussure, 1877 (type locality: Japan, Celebes).

Diagnosis. Body coloration dark brown, appendages more brightly mottled. Sexual dimorphism remarkable: male with enlarged round head and full winged tegmina, but female with relatively normal head and short tegmina. Head with transverse strip between lateral ocelli, but sometimes not noticeable. Hind wings degenerated, not surpassing tegmina. Fore tibia with only outer tympanum. Male epiphallus with a pair of prominent lateral lobes and a wide median lobe.

Remarks. It should be noted that Randell (1964) designated Velarifictorus micado as the type species of this genus, but its figures and genitalic descriptions are actually misidentified Velarifictorus aspersus, as mentioned by Ichikawa et al. (2000), and Ichikawa (2001).

\section{Subgenus Velarifictorus Randell, 1964}

Type species: Scapsipedus micado Saussure, 1877 (type locality: Japan, Celebes). 
Diagnosis. Male epiphallus with relatively long, but not high hind median projection directed backwards. The other two subgenera diagnoses are as follows: Buangina from Australia, male epiphallus with long and high hind median projection; Pseudocoiblemmus from Indochina, male epiphallus with long, but small hind median projection (Gorochov, 2001).

Remarks. All known Korean members belong to the nominate subgenus.

\section{1 *Velarifictorus aspersus borealis Gorochov, 1985} (Tables 1, 2, Figs. 1A, B, 2A, 3A, 4A, 5A)

Velarifictorus aspersus borealis Gorochov, 1985: 91 (type locality: China, Beijing); Storozhenko and Paik 2007: 100; Paik et al., 2010: 78.

Gryllus aspersus Walker, 1869: 39 (type locality: Hongkong); Scapsipedus aspersus: Wu, 1935: 70; Cho, 1959: 144; 1969: 769; Choo and Choi, 1983: 49; Song, 1988: 357; 1989: 283; Bae and Lee, 1990a: 59; 1990b: 67; Velarifictorus aspersus: Kwon and Huh, 1994: 49; Moon and Yoon, 1994: 106; Kwon et al., 1996: 104; Hua 2000: 56; Paek et al., 2010: 35; Kim, 2011: 65.

=Gryllodes berthellus Saussure, 1877: 205 (type locality: Japan, Amboine, Banka, China); Shiraki, 1930: 218 ("Keijo" =Seoul); Doi, 1932: 38; Ju, 1993: 286; Gryllodinus berthelles [!]: Ju, 1969: 19; Gryllodes berhellus [!]: Hua, 2000: 54 [a synonym by Chopard, 1967].

=Scapsipedus mandibularis Saussure, 1877: 246 (type locality: India, Bombay, Japan); Doi, 1932: 38; Cho, 1959: 145; 1969: 769; Bae and Lee, 1990a: 59; 1990b: 67; Kwon and Huh, 1994: 49; Moon and Yoon, 1994: 106; Kwon et al., 1996: 104; Scapsipedlus [!] mandibularis: Ju, 1969: 21; Velarifictorus mandibularis: Hua, 2000: 57 [a synonym by Chopard, 1967].

Material examined (50 specimens). Korea: Gyeongsangnam-do: 1 万ٓ (light trap), Jinju-si, Yugok-dong, Nabul-ri,

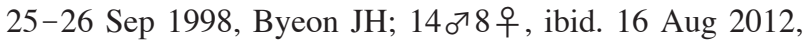
Kim TW, Kim OS; 1 지 1 우, Sacheon-si, Chukdong-myeon, Baechun-ri, 17 Aug 2012, Kim TW; $1 \sigma^{\top}$, Tongyeong-si, Jeongryang-dong, 8 Oct 2011, Sung KS; $2 \sigma^{\top}$, Tongyeong-si, Inpyeong-dong, Gyeongsang National University College of Marine Science Campus, 23 Aug 2006, Jang YK; 5 이 8 우, ibid. 17 Aug 2012, Kim TW, Kim OS; Jeollanam-do: 5 శð 3 우, Yeosu-si, Sangam-dong Sangam elementary school, Mt. Yeongchuisan, 28 Aug 2012, Kim OS; Japan: $1 \sigma^{\nearrow}$, Kyoto Prefecture, Kyoto City, Sakyo-ku, Yoshida, Mt. Yoshidayama, alt. 100-120 m, 21 Sep 1988, S. Fujii.

Description. Male (Fig. 1A): Head globulous, sufficiently wider than anterior margin of pronotum in dorsal view; ver- tex transversely dark brown; occiput bright brown with six longitudinal pale strips posteriorly; a distinct strip transverse between lateral ocelli, its length two times wider than horizontal diameter of antennal socket; median ocellus located between inner upper margins of antennal sockets, surrounded with a short bright line linked downwardly; subocular area weakly depressed, coarsely furrowed; mandibles elongated, thurs clypeal suture transverse higher than mid in head, the suture thickened in upper margins, but diminished in mid; scape as long as wide; palpi bright. Pronotum a little shorter than fore femur, two times wider than long in dorsal view; anterior margin slightly wider than posterior one, both truncated, pilose; lateral lobe of pronotum slightly deeper than pronotal length, lower half area bright. Tegmina (Fig. 3A) rather wider than posterior margin of pronotum, not reaching abdominal end; harp veins two; chordal veins three; mirror area divided, 1.2 times wider than long; apical area well netted, as long as mirror area; lateral field deeper than lateral lobe of pronotum, with 5-6 longitudinal veins. Fore tibia with largely oval-shaped outer tympanum, 3.5 times longer than wide; inner tympanum vestigial, not discernable. Hind femur 2.7 times longer than middle femur. Hind tibia with five pairs of large dorsal spines on both sides, the largest inner apical spur as long as half of basitarsus. Hind barsitarsus two times longer than remaining two tarsomeres combined length; six dorsal spinules on both sides; inner apical spur as long as last tarsomere. Cercus as long as three fifths of hind femur. Subgenital plate as long as half of basal width, its hind margin weakly notched in mid.

Genitalia (Fig. 4A): Epiphallus with a broad and short median lobe, but which is rather distinctly divided posteriorly as a pair of small obtuse-angular lobes by median concavity; lateral lobes longer than middle lobe, with long hairs. Ectoparamere greatly projected beyond hind margin of epiphallus, its basal half rather thickened, but its distal half slightly incurved and sharply tapered at apex, hook-shaped.

Calling sound (Fig. 5A): Shortly and slowly repeated song phrase, each consisting of 1-3 distinctive chirps; their intervals sometimes irregular depending on outer conditions, when regularly repeated mean chirps two; a chirp with undulating 5-8 syllables at $29^{\circ}$ condition; each syllable lasted $0.03 \mathrm{~s}$; dominant frequency spectrum $3.5-5 \mathrm{KHz}$.

Female (Fig. 1B): Head smaller than in male, mandibles moderate, not enlarged. Tegmina shorter than male, just covering two thirds of dorsal abdomen, its apex roundly truncated; medial area longitudinally bright. Subgenital plate convex, roundly trapezoidal, its hind margin narrowly truncated. Ovipositor as long as hind femora.

Distribution. Velarifictorus aspersus borealis: Korea, Japan, 
North China; Velarifictorus aspersus aspersus: South China, Taiwan, Thailand, Bhutan, India, Sri Lanka, Malaysia, Myanmar, Singapore, Indonesia (widely distributed in South East Asia, Oriental region).

Remarks. Although there were plentiful records of Velarifictorus aspersus in Korea, examined materials were only of four male specimens before the year 2012, from the far southern province Gyeongsangnam-do. Through recent field trips attained in 2012 by myself and my colleague Mr. Kim Oh-Sung, the cricket was recollected from the same locality, and a few neighboring localities added in Gyeongsangnamdo. The other specimens were also collected from the far southern province Jeollanam-do by Mr. Kim Oh-Sung in the same year. Therefore, its distributional ranges seem to be rather scattered, but quite restricted, only in far southern areas. The burrowing cricket is, however, never as common a cricket as Velarifictorus micado in Korea. Probably, earlier records of Velarifictorus aspersus are confused with misidentifications, or just citing repetition without taxonomic consideration. Besides, Storozhenko and Paik (2007) introduced subspecies Velarifictorus aspersus borealis for northern populations, based on their nominotypical distributions i.e. borealis occupies north (type locality: Beijing) against aspersus occupies south (type locality: Hongkong). Gorochov (1985, figs. 1-4) described borealis as rather bracypterous and male epiphallus with deeply emarginated hind margin against aspersus with male epiphallus shallowly emarginated hind margin. As compared with Korean materials (Fig. 4A), the structure of male epiphallus more close to borealis rather than aspersus, which subspecific consideration is agreed herein.

\section{${ }^{1 *}$ Velarifictorus (Velarifictorus) micado (Saussure, 1877) (Tables 1, 2, Figs. 1C, D, 2B, 3B, 4B, 5B)}

Scapsipedus micado Saussure, 1877: 247 (type locality: Japan, Celebes); Velarifictorus micado: Kwon and Huh, 1994: 50; Kwon et al., 1996: 104; Paik et al., 2010: 30, figs. 36ab; Kim, 2011: 67; Velarifictorus mikado [!]: Storozhenko and Paik, 2007: 100; Paek et al., 2010: 35.

Material examined (147 specimens). Korea: Gangwon-do: 1 지우 (light trap), Gangneung-si, Samsan-ri, Buyeongyegok Valley, 18-19 Aug 2001, Kim JI, Kim SY; 1 ㄲ 5 우, Gangneung-si, Okgye-myeon, Sangye-ri, Mt. Seokbyeongsan, 27-28 Aug 2002, Kim TW; 1 乃3 우, Yeongwol-gun, Gonggiri, Mt. Sambangsan, 17-18 Sep 2001, Kim TW, Kim MA; 1 우 (light trap), Yeongwol-gun, Mt. Baekdeoksan, Gwaneumsa Temple, 26 Aug 2001, Kim SY, Kim AY; 1 우, Jeongseongun, Nam-myeon, Jeungsan, 19 Sep 2004, Kim TW; 3 우,
Chuncheon-si, Gangchon, 20 Sep 1996, Huh R; $1 \sigma^{7}$, Hwacheon-gun, Haesanryeong Pass, 3 Oct 2006, Kim TW; 1 ऽ , Cheongsong-gun, Chongpyeong, 16 Aug 1988, Oh HJ; 1 우, Wonju-si, Haenggu-dong, Mt. Chiaksan, Hwanggol Office, 1 Sep 2006, Kang TH; Gyeonggi-do: 5 지 6 우 (pitfall), Gapyeong-gun, Mt. Cheonggyesan, 27 Sep 2002, Jeong MP; $1 \sigma^{\nearrow}$,

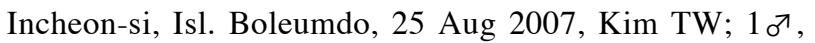
Goyang-gun, Madu-dong, 14 Aug 1994, Park SK; $1 \sigma^{\nearrow}$, Goyang-gun, Hwajeong-dong, 1 Sep 2009, Kim TW; 4 ㄲ 1 우, Yeoncheon-gun, Jeonguk-eup, Hantangang River-bed, 23 Sep 2011, Kim TW; 1 万 , Pocheon-gun, Gwangneung Arboretum, 12 Sep 1991, Jeong SM; 1 우, ibid. 6 Aug 1992, Park SJ; 1 우, Gwangju-si, Eommi-ri, 15 Sep 1984, Kim JI; 1 ㄲ, Gunpo-si, Mt. Surisan, 7 Oct 2001, Kim TW; 1 이 1 우, Pajusi, Mt. Gamaksan, 8 Sep 1984, Kim JI; 1 우, Namyangju-gun, Mt. Cheonmasan, 21 Sep 1987, Kim MS; 1 우, Suwon-si, Cheongcheon-dong, 9 Oct 1987, Lee JH; 2 우, Ansan-si, Daebudo, Sihwaho Lake, 27 Aug 2004, Kim TW; 2 주우 (pitfall), Ansan-si, Singil-cheon, 19 Oct 2002, Jeong MP; 6 ㄱ7 우 (pitfall), Hanam-si, Misa-dong, 28 Sep 2002, Jeong MP; 1 우, Uijeongbu-si, Gosan-dong, 23 Aug 1992, Choi EA; 1 우, Mt. Bulamsan, 18 Sep 1982, Lee MK; 1 우, Yangpyeong-gun, Yeonsu-ri, 31 Aug 1980, Chang KS; Seoul: 2 지우, Eun-

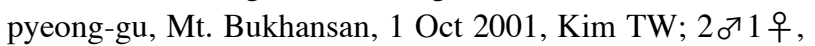
ibid. 3 Oct 2003, Kim TW; 2 우, Bukhansanseong, 26 Sep 1991, Jeong SM; 1 우, Jungryang-gu, Junghwa-dong, 12 Sep 1988, Sim HJ; 1 우, Jungryang-gu, Mt. Mangwusan, 4 Oct 1996, Lee SY; 1 우, Uenpyeong-gu, Yeokchon-dong, 9 Oct 1987, Lee MK; 1 우, Dongjak-gu, Sangdo-dong, 4 Aug 1985, Han SO; 1 우, Gangnam-gu, Mt. Cheonggyesan, 14 Aug 1985, Yoon HJ; $1 \sigma^{\top}$, Mt. Kachisan, 25 Aug 1982, Lee EK; 2 ð, Mt. Gwanaksan, 25 Sep 1982, Yoo YS, Yoo HK; 1 ð, ibid. 8 Sep 1990, Seo MJ; 1 ð , Seocho-gu, Mt. Woomyeonsan, 1 Sep 1991, Oh JM; 1 우, Wolgye-dong, 6 Nov 1982, Park HM; 1 우, Seongbuk-gu, Dongseon-dong, 30 Sep 1997, Cho KH; 1 ð, Mok 3-dong, 22 Aug 1996, Jeong EJ; 1 ð, Dobong-gu, Sinchang-dong, 24 Aug 1987, Park K; Chungcheongbuk-do: 1 우 (light trap), Jecheon-si, Deoksan-myeon, Dogi-ri, Mt. Wolaksan, Munsubong, 14-15 Sep 1996, Jeon JS; 3 우, Chungju-si, Sancheok-myeon, Mt. Cheondeungsan, 28 Aug 2002, Kim TW; 2 우, Chungju-si, Jongmin-dong, Mt. Gyemyeongsan, 29 Aug 2002, Kim TW; $4 \sigma^{\top 1} 1$ 우, Chungjusi, Gageum-myeon, Chungju Expressway Resting Place (a down line), 25 Aug 2011, Kim TW; Chungcheongnam-do: 1 우, Gongju-si, Jakgol, 8 Aug 1982, Kim HK; 1 우, Geumsangun, Chubu-myeon, Sundang-ri, Mt. Seodaesan, 28 Sep 2002, Kim AY; $1 \sigma^{\nearrow}$, Hongseong-gun, Gwangcheon-eup, 19 Sep 1998, Yoo JS; Gyeongsangbuk-do: 1 우, Daegu-si, Nam-gu, Daemyeong-dong, 18 Aug 1995, Park EM; Gyeongsangnam-

Korean name: ${ }^{1 *}$ 극동귀뚜라미 
do: 1 우 (light trap), Goseong-gun, Sangri-myeon, Mt. Muisan, Munsuam, 14-15 Oct 1999, An TH; 1 万ٓ (light trap), Namhae-gun, Simcheon-ri, Simcheoncheon, 29-30 Aug 1998, Jeon JS; $2 \sigma^{7} 1$ 우, Sacheon-si, Chukdong-myeon, Baechunri, 13 Sep 2001, Paik YH; 1 『 (light trap), Sacheon-si, Waryong-dong, Mt. Waryongsan, Cheongryongsa, 5-6 Oct 1996, An TH; 1 우 (light trap), Jinju-si, Naedong-myeon, Sinyul-ri,

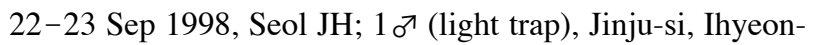
dong, Mt. Sukhosan, 23-24 Sep 1998, Jeon JS; 1 ㄲ2우, Jinju-si, Yugok-dong, Nabul-ri, 16 Aug 2012, Kim TW, Kim OS; 1 万つ, Tongyeong-si, Jeongryang-dong, 8 Oct 2011, Sung $\mathrm{KS} ; 1$ 우, Hadong-gun, Hwagae-myeon, Ssanggyesa Temple,

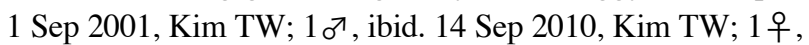
Busan-si, Yeongdo-gu, Cheongsan APT, 30 Aug 2000, Kim SK; 1 기 4 우, Haman-gun, Mt. Yuhangsan, 21-23 Sep 2006, Lee DH, Park JH, Park YN; 1 우, Busan-si, Gijang-gun, Ilgwang-myeon, Won-ri, Mt. Dareum, 20 Sep 2008, Sang HS; 1 우, Busan-si, Gijang-gun, Gijang-eup, Mt. Samgak, 19 Sep 2008, Moon TY; Jeollabuk-do: 3 이 1 우 (pitfall), Buan-gun, Haseo-myeon, Haechang-ri, 24 Sep 2001, Jeong MP; Jeollanam-do: 1 우, Mokpo-si, Sanjeong-dong, Samhakdo, 16 Oct 2004, Kim TW; 1 이눙, Gwangyang-si, Dapgok, Mt. Baekunsan, 15 Sep 2001, Paik JC; 1 ㄲ1우, Suncheon-si, Mae-

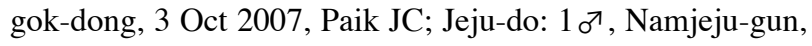
Andeok-myeon, Sagye-ri, Mt. Sanbangsan, 14 Aug 2004, Kim JK, Kim TW; 1 万 , Namjeju-gun, Andeok-myeon, Andeokgyegok Valley, 15 Oct 2008, Kim TW; $1 \sigma^{\top}$, Jeju-si, Nohyeong-dong, 7 Oct 2006, Jung SH; $1 \sigma^{\top}$, Seoguipo-si, Cheonjiyeon-pokpo, 10 Oct 2011, Kim TW.

Description. Male (Fig. 1C): Head globular, slightly wider than anterior margin of pronotum; vertex roundly merging with frons; occiput with six pale longitudinal strips posteriorly; a distinct strip transverse between lateral ocelli, merging with $\wedge$-shaped ecdysial line, its length two times wider than horizontal diameter of antennal socket; median ocellus located between inner upper margins of antennal sockets, surrounded by a bright line linked downwardly; subocular area weakly depressed, coarsely punctured; mandibles vertical; clypeal suture transverse lower than mid in head; scape as long as wide; palpi bright. Pronotum as long as fore femur, two times wider than long in dorsal view; anterior and posterior margins truncated, pilose; lateral lobe of pronotum as deep as pronotal length, lower margin bright. Tegmina (Fig. 3B) slightly wider than hind margin of pronotum, not reaching abdominal end; harp veins two; chordal veins tree; mirror area divided, as long as wide; apical area well netted, slightly shorter than mirror area; lateral field deeper than lateral lobe of pronotum, with 6-7 longitudinal veins. Fore tibia with largely oval-shaped outer tympanum, its length four times longer than wide; inner tympanum vestigial, not discernable. Hind femur three times longer than middle femur. Hind tibia with five pairs of large dorsal spines on both sides, the largest inner apical spur as long as a half of basitarsus. Hind barsitarsus two times longer than remaining two tarsomeres combined length; 5-6 dorsal spinules on both sides; inner apical spur as long as two thirds of last tarsomere. Cercus as long as three fifths of hind femur. Subgenital plate as long as half of basal width, its hind margin weakly notched in mid.

Genitalia (Fig. 4B): Epiphallus with a moderate-sized median lobe, its hind margin rather transversely truncated, a small emargination present on mid posteriorly, but sometimes not discernable; lateral lobes longer than middle lobe, with long hairs. Ectoparamere acute blade-shaped, greatly projected surpassing hind margin of epiphallus, its apex triangularly pointed.

Calling sound (Fig. 5B): Continuously lasting song phrase, distinctive chirps 4-5 times regularly repeated for $1 \mathrm{~s}$; a chirp with undulating $6-8$ syllables at $27^{\circ}$ condition; each syllable lasted $0.02 \mathrm{~s}$; dominant frequency spectrum 3-4 KHz.

Female (Fig. 1D): Head slightly smaller than in male, without subocular depression. Tegmina shorter than male, just covering two thirds of abdomen, its apex narrowly rounded; medial area longitudinally bright. Subgenital plate convex, roundly triangular, its hind margin narrowly truncated. Ovipositor 1.5 times longer than hind femora.

Distribution. Korea, Japan, China, Vietnam, Sulawesi; North America (introduced).

Remarks. This burrowing cricket is the most widespread Velarifictorus in the Korean peninsula based on the distributional data, although its citing data are fewer than Velarifictorus aspersus. Its distributional ranges extend towards the north to Far Eastern Russia (Storozhenko, 2004), across North Korea (Kim and Puskás, 2012). The male actively singing between summer and autumn seasons, even where near human residence or city parks. It is also culturally well known as Chinese fighting crickets (Jin, 1994).

${ }^{1 *}$ Velarifictorus (Velarifictorus) ornatus (Shiraki, 1911) (Tables 1, 2, Figs. 1E, F, 2C, 3C, 4C, 5C)

Gryllus ornatus Shiraki, 1911: 52, Pl. 1, fig. 4A, B (type locality: "Formosa"=Taiwan); Velarifictorus ornatus: Yin and Liu, 1995: 62 (China); Ichikawa et al., 2000; 268 (Japan).

Material examined (38 specimens). Korea: Gyeonggi-do: 1 우, Gwangju-si, Gwangju-eup, 13 Jun 1992, Shin MJ; 1 ऽ, Gunpo-si, Mt. Surisan, 19 Jun 2005, Kim TW; 1 우, Yonginsi, Giheung, 31 May 2003, Choi JE; 1 우, Incheon-si, Ongjin-

Korean name: ${ }^{1 *}$ 봄여름귀뚜라미 (신칭) 
Table 1. Differential characters of Velarifictorus and Lepidogryllus in Korea

\begin{tabular}{|c|c|c|c|c|}
\hline Character & $\begin{array}{l}\text { Velarifictorus aspersus } \\
\text { borealis }\end{array}$ & Velarifictorus micado & Velarifictorus ornatus & Lepidogryllus siamensis \\
\hline $\begin{array}{l}\text { Transverse strip } \\
\text { between lateral } \\
\text { ocelli }\end{array}$ & Present & Present & Indistinct & Present \\
\hline Mandibles of males & Prolonged & Moderate & Moderate & Normal \\
\hline Tegmina of males & $\begin{array}{l}\text { Apical area as long as } \\
\text { mirror area }\end{array}$ & $\begin{array}{c}\text { Apical area shorter } \\
\text { than mirror area }\end{array}$ & $\begin{array}{l}\text { Apical area shorter } \\
\text { than mirror area }\end{array}$ & $\begin{array}{r}\text { Apical area longer } \\
\text { than mirror area }\end{array}$ \\
\hline Genitalia of males & $\begin{array}{l}\text { Epiphallus with } \\
\text { median lobe }\end{array}$ & $\begin{array}{l}\text { Epiphallus with } \\
\text { median lobe }\end{array}$ & $\begin{array}{l}\text { Epiphallus with } \\
\text { median lobe }\end{array}$ & $\begin{array}{l}\text { Epiphallus without } \\
\text { median lobe }\end{array}$ \\
\hline Ovipositor & Shorter than hind femur & Longer than hind femur & As long as hind femur & As long as hind femur \\
\hline Voltinism & $\begin{array}{l}\text { Univoltine, } \\
\text { overwinter as egg }\end{array}$ & $\begin{array}{l}\text { Univoltine, } \\
\text { overwinter as egg }\end{array}$ & $\begin{array}{l}\text { Univoltine, } \\
\text { overwinter as nymph }\end{array}$ & $\begin{array}{l}\text { Bivoltine, } \\
\text { overwinter as nymph }\end{array}$ \\
\hline Active season & $\begin{array}{l}\text { Summer to autumn } \\
\text { (Aug-Oct) }\end{array}$ & $\begin{array}{l}\text { Summer to autumn } \\
\text { (Aug-Oct) }\end{array}$ & $\begin{array}{l}\text { Spring to summer } \\
\text { (late May-Aug) }\end{array}$ & $\begin{array}{l}\text { Spring to autumn } \\
\text { (May-Sep) }\end{array}$ \\
\hline
\end{tabular}

Table 2. Measurements of Velarifictorus and Lepidogryllus in Korea (length in $\mathrm{mm}$ )

\begin{tabular}{|c|c|c|c|c|c|}
\hline Species & Body & Pronotum & Tegmen & Hid femur & Ovipositor \\
\hline $\begin{array}{l}\text { Velarifictorus aspersus } \\
\text { borealis }\end{array}$ & $\begin{array}{ll}\text { 이 } 14.1-20.1 \\
\text { 우 } 13.2-16.9\end{array}$ & $\begin{array}{l}\text { 가 } 2.2-2.8 \\
\text { 우 } 2.7-3.1\end{array}$ & $\begin{array}{l}\text { 가 } 7.8-8.8 \\
\text { 우 } 6.6-7.8\end{array}$ & $\begin{array}{l}\text { 가 } 8.6-11.2 \\
\text { 우 } 8.9-10.8\end{array}$ & 우 7.8-9.1 \\
\hline Velarifictorus micado & $\begin{array}{l}\text { 주 } 11.7-18.9 \\
\text { 우 18.0-22.0 }\end{array}$ & $\begin{array}{ll}\text { 가 } 2.1-2.8 \\
\text { 우 } 3.1-3.4\end{array}$ & $\begin{array}{l}\text { 자 7.8-9.9 } \\
\text { 우 5.5-10.3 }\end{array}$ & $\begin{array}{l}\text { 저 } 9.2-11.4 \\
\text { 우 10.0-11.5 }\end{array}$ & 우 11.7-14.1 \\
\hline Velarifictorus ornatus & $\begin{array}{l}\text { 각 } 11.8-15.6 \\
\text { 우 } 11.5-12.0\end{array}$ & $\begin{array}{l}\text { 가 } 2.3-2.8 \\
\text { 우 } 2.5-2.7\end{array}$ & $\begin{array}{l}\text { 가 } 6.7-8.2 \\
\text { 우 } 4.6-8.3\end{array}$ & $\begin{array}{l}\text { 가 } 7.4-9.4 \\
\text { 우 } 8.3-9.0\end{array}$ & 우 7.7-9.6 \\
\hline Lepidogryllus siamensis & $\begin{array}{l}\text { 저 } 14.6-17.5 \\
\text { 우 14.0-15.2 }\end{array}$ & $\begin{array}{l}\text { 가 } 2.5-3.2 \\
\text { 우 } 2.5-3.0\end{array}$ & $\begin{array}{l}\text { 주 8.6-10.2 } \\
\text { 우 8.7-9.9 }\end{array}$ & $\begin{array}{l}\text { 가 } 8.7-9.7 \\
\text { ㅇ } 8.9-9.7\end{array}$ & 우 8.6-9.5 \\
\hline
\end{tabular}

gun, Deokjeok-myeon, Seopo-ri, 14 Jun 1962, Na YJ; 1 우,

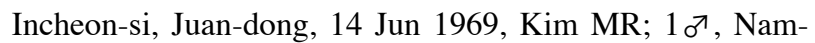
yangju-si, Mt. Cheonmasan, 14 Jun 1979, Kim TS; 2 우, Hwaseong-si, Jangan-myeon, Bangchukmal, 16 Jul 2009, Park YK; 2 우, Paju-si, Beopwon-eup, 6 Jul 2008, Kim TW; 1 万, Paju-si, Gwangtan-myeon, Bogwangsa Temple, 9 Jun 1979, Kim SH; Seoul: 1 우, Daebang-dong, 31 May 1963, Kim HJ; 1 우, Seongbuk-gu, Jeongneung, 21 Jun 1959, Kim YI; 1 기, ibid. 13 Jun 1959, Lee SA; 1 우, Sinchon, 26 May 1965, Shin SJ; 1 ð , Eunpyeng-gu, Bulgwang-dong, 28 Jun 1960, Hong HJ; Chungcheongnam-do: $2 \sigma^{\top 3}$ 우, Seosan-si, Haemi-myeon, Hanseo University Campus, Mt. Gayasan, 22 Jun 2001, Kim TW; Gyeongsangbuk-do: 1 乃 , Sangju-si, 23 Jun 2000, Park JK; Gyeongsangnam-do: 1 우 (light trap), Goseong-gun, Sangri-myeon, Yongtae-ri, Mt. Sanseongsan, 10-11 Aug 1996, Jeon JS; 1 지우우, Hadong-gun, Hwagae-myeon, Mt. Jirisan, Ssanggyesa Temple, 31 Jun 2001, Kim TW; 2 우, Geoje-si, Yeoncho-myeon, Songjeong-ri, 11 Jun 2009, Kim TW; 1 우 (sweeping), Yangsan-si, Mt. Wonhyosan, 28 Aug 2002, Do YH; Jeollanam-do: 3 万๐, Wando-gun, Wando-eup, Wando Tower, 1 Jul 2010, Kim TW; 1 ð, ibid. 19 Jul 2012, Kim
TW; 2 ○ $^{\top} 1$ 우, Wando-gun, Cheongsan-myeon, Yeoseo-ri, 20 Jul 2012, Kim TW; 1 우, Gokseong-gun, 7 Jul 1999, Paik YH; 1 万, Haenam-gun, Munnae-myeon, Jindo Bridge, 16 Jun 2011, Sung KS; $1 \sigma^{\nearrow}$, Jindo-gun, Dongoe-ri, Mt. Suribong, N34 ${ }^{\circ} 28^{\prime} 37.8^{\prime \prime}$, E126 $18^{\prime} 04.1^{\prime \prime}$, alt. 183 m, 29 Jun 2004, Sohn JC, Park HJ, Nam SC, Han YE.

Description. Male (Fig. 1E): Head globular, slightly wider than anterior margin of pronotum; vertex and frons roundly merging; occiput with six pale longitudinal strips posteriorly; a transverse strip absent between lateral ocelli, diminished to feeble ecdysial line, thurs hardly distinguished; median ocellus located between inner upper margins of antennal socket, linked with short bright line downwardly; subocular area indistinctly furrowed; mandibles vertical; clypeal suture transverse lower than mid in head; scape as long as wide; palpi bright. Pronotum as long as fore femur, two times wider than long in dorsal view, with a pair of dorsal crescent patterns; anterior and posterior margins truncated, pilose; lateral lobe of pronotum as deep as pronotal length, lower half area bright. Tegmina (Fig. 3C) as wide as hind margin of pronotum, not reaching abdominal end; harp veins two; chordal 

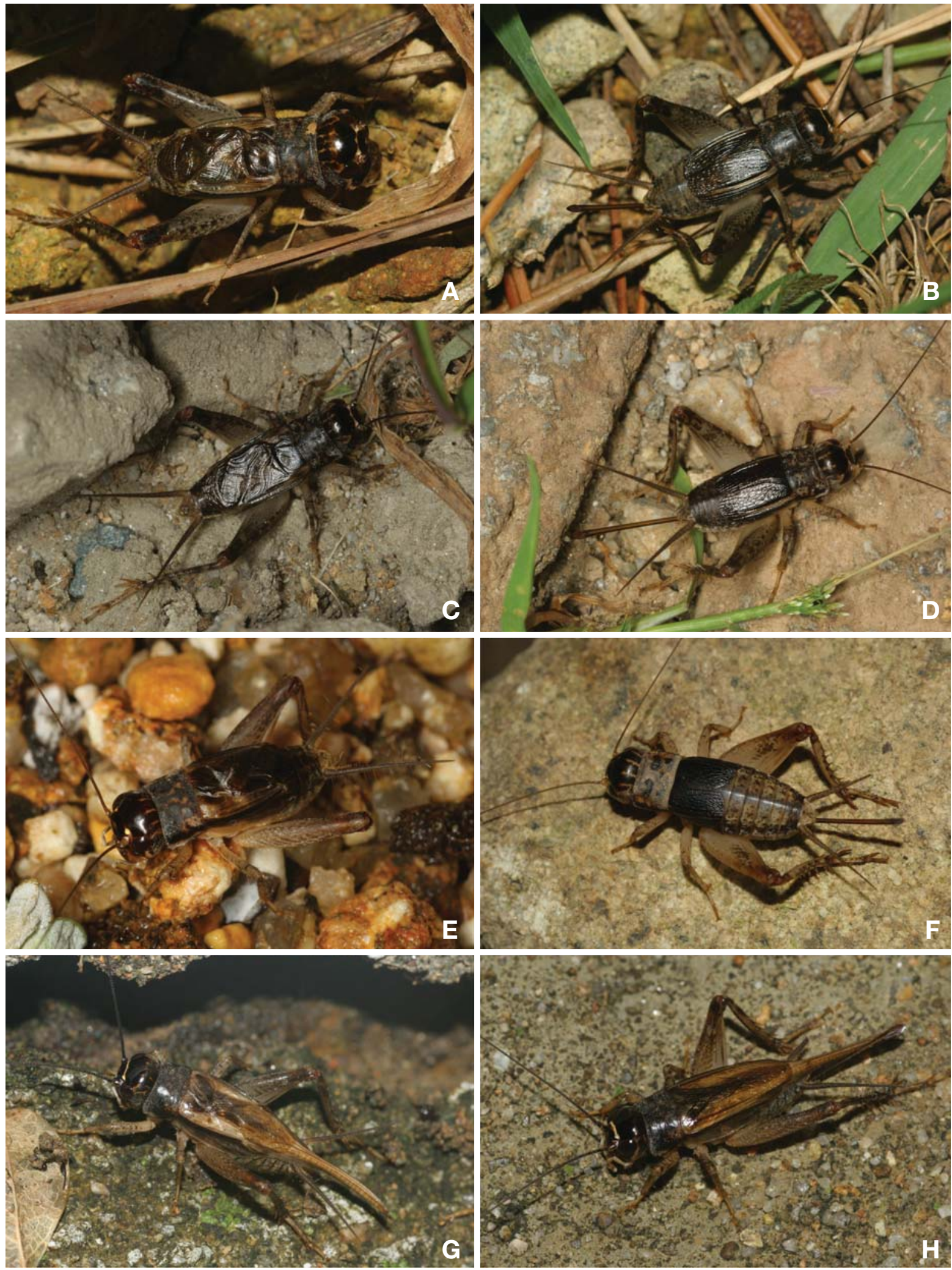

Fig. 1. Habitus of Velarifictorus and Lepidogryllus from Korea (A, C, E, G, males; B, D, F, H, females). A, B, Velarifictorus aspersus borealis; C, D, Velarifictorus micado; E, F, Velarifictorus ornatus; G, H, Lepidogryllus siamensis. 

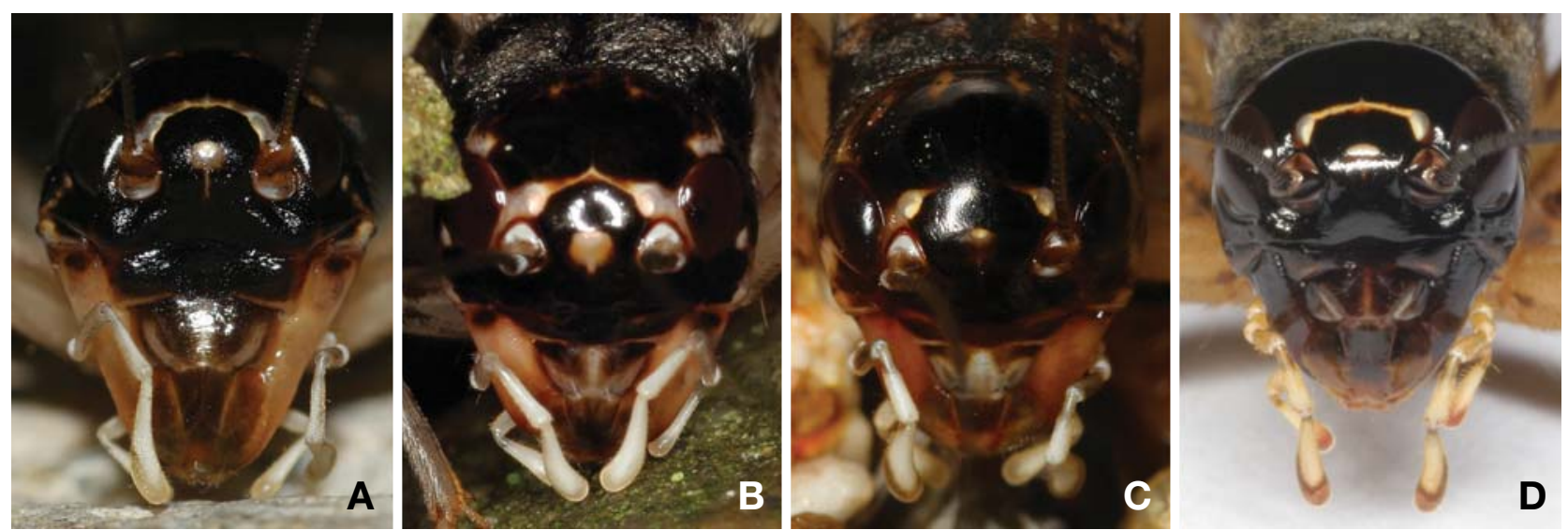

Fig. 2. Male's head of Velarifictorus and Lepidogryllus from Korea. A, Velarifictorus aspersus borealis; B, Velarifictorus micado; C, Velarifictorus ornatus; D, Lepidogryllus siamensis.

veins three; mirror area divided, as long as wide; apical area well netted, as long as two thirds of mirror area; lateral field deeper than lateral lobe of pronotum, with 5-6 longitudinal veins. Fore tibia with largely oval-shaped outer tympanum, its length three times longer than wide; inner tympanum vestigial, not discernable. Hind femur 2.7 times longer than middle femur. Hind tibia with five pairs of large dorsal spines on both sides, the largest inner apical spur as long as a half of basitarsus. Hind barsitarsus 1.7 times longer than remaining two tarsomeres combined length; 5-6 dorsal spinules on both sides, inner apical spur as long as two thirds of last tarsomere. Cercus as long as thee fifths of hind femur. Subgenital plate as long as half of basal width, its hind margin weakly notched in mid.

Genitalia (Fig. 4C): Epiphallus with relatively small and short median lobe, its hind margin transversely truncated, trapezoidal; lateral lobes longer than middle lobe, with long hairs. Ectoparamere slightly projected beyond hind margin of epiphallus, its basal portion rather thickly elongated, but its distal portion sharply pointed and abruptly incurved at apex.

Calling sound (Fig. 5C): Slowly and regularly repeated song phrase; one chirp for one second, but their intervals sometimes irregularly ceased, depending on outer conditions; a chirp lasted $0.45-0.5 \mathrm{~s}$ with $46-54$ dense syllables, whereas interlude between the chirps taken $0.5-0.55 \mathrm{~s}$ at $28^{\circ}$ condition; dominant frequency spectrum $4.3-6.2 \mathrm{KHz}$.

Female (Fig. 1F): Head size similar to male, but tegmina shorter than male, just covering less than half of dorsal abdomen, its apex roundly truncated. Subgenital plate convex, roundly trapezoidal, its hind margin narrowly truncated. Ovipositor as long as hind femora.
Distribution. Korea (new record), Japan, China, Taiwan. Remarks. This burrowing cricket overwinters as nymphal stages. Calling sounds of the adult can be heard seasonally more earlier than the other Velarifictorus between late May and mid August (Table 1).

\section{Genus Lepidogryllus Otte and Alexander, 1983}

Type species: Gryllus parvulus Walker, 1869 (type locality: South Australia).

Diagnosis. Body coloration dark brown, legs brighter than body, mottled with speckles. Sexual dimorphism not more remarkable than Velarifictorus: head globose in both sexes, as wide as frontal margin of pronotum, frontal rostrum slightly projecting, mandibles normal. Head with white transverse strip between lateral ocelli. Tegmina fully winged in both sexes, hind wings largely surpassing tegmina, sometimes absent (autotomy). Interspace between the antennal sockets wider than width of themselves, clypeal suture transverse. Fore tibia with large outer and small inner tympana. Male epiphallus wide U-shaped, with a pair of prominent lateral lobes, but without median lobe.

\section{${ }^{1 *}$ Lepidogryllus siamensis (Chopard, 1961) com. \& stat. nov. (Tables 1, 2, Figs. 1G, H, 2D, 3D, 4D, 5D)}

Modicogryllus siamensis Chopard, 1961: 280, P1. 7 (type locality: "Siam"=Thailand); Paik et al., 2010: 77; Paek et al., 2010: 34; Kim, 2011: 49.

Scapsipedus parvus nec Chopard, 1928: 18 (type locality: India); Scapsipedus parves [!]: Choo and Choi, 1983: 49; Velarifictorus parvus: Kwon and Huh, 1994: 50; Kwon et al., 1996: 104.

Korean name: ${ }^{1 *}$ 샴귀뚜라미 (신칭) 


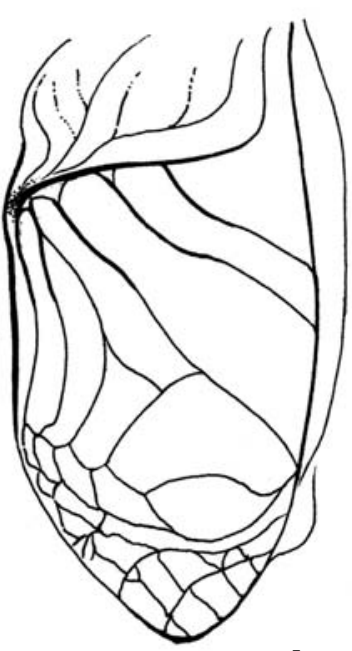

A

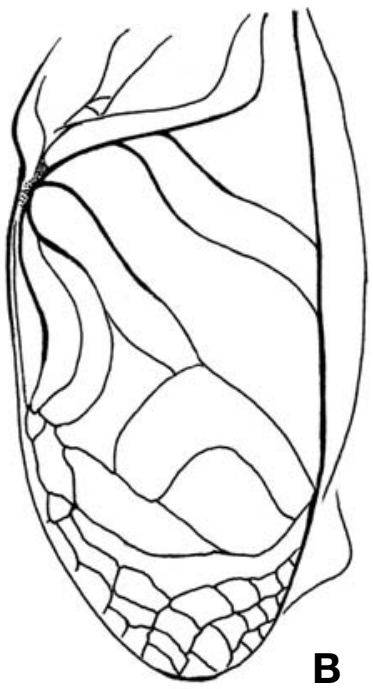

B
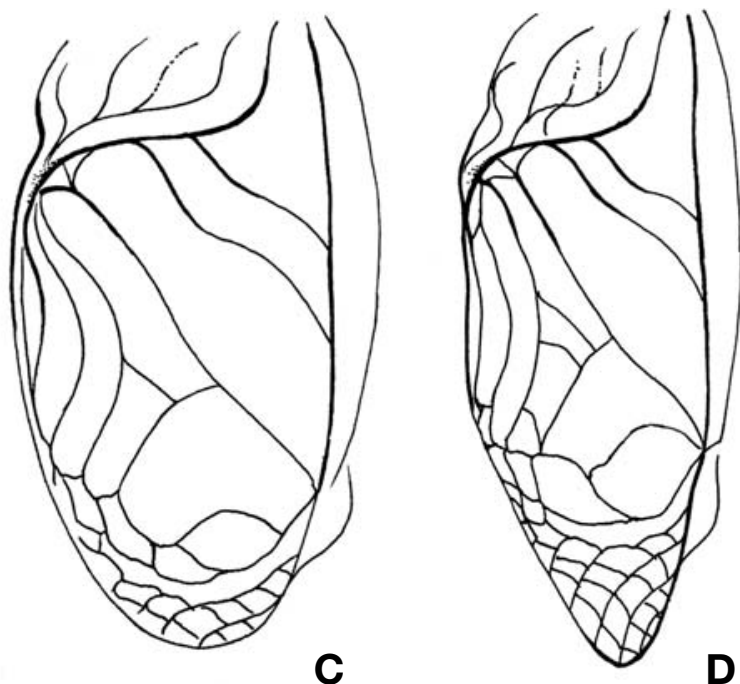

Fig. 3. Male's tegmina of Velarifictorus and Lepidogryllus from Korea. A, Velarifictorus aspersus borealis; B, Velarifictorus micado; C, Velarifictorus ornatus; D, Lepidogryllus siamensis.

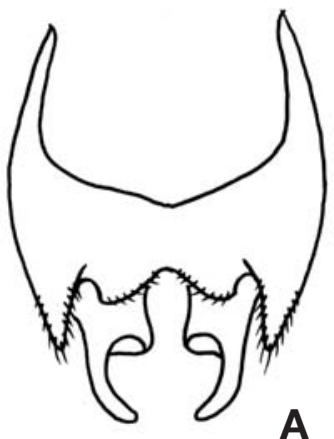

A

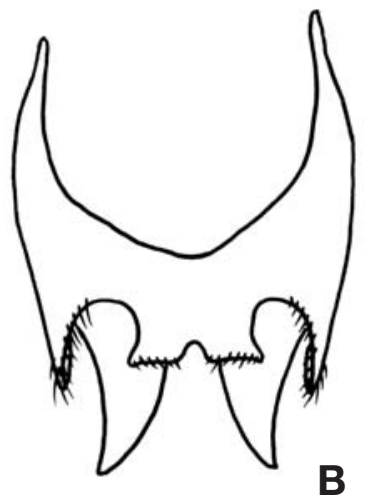

B
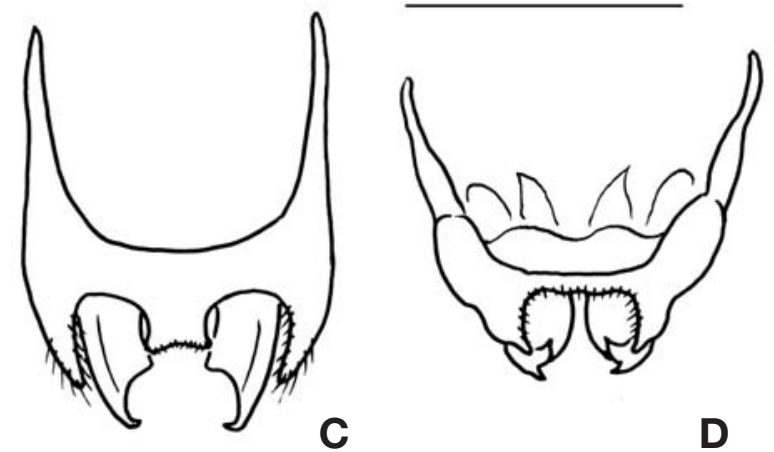

C

D

Fig. 4. Male genitalia of Velarifictorus and Lepidogryllus from Korea (dorsal view). A, Velarifictorus aspersus borealis; B, Velarifictorus micado; C, Velarifictorus ornatus; D, Lepidogryllus siamensis. Scale bar $=1.0 \mathrm{~mm}$.

Material examined (58 specimens). Korea: Chungcheongbuk-do: 1 우 (light trap), Danyang-si, 14-15 Jun 1993, Anonym; Gyeongsangbuk-do: 1 우, Uljin-gun, Sokwang-ri, 1 Aug 1999, Ku DS; Gyeongsangnam-do: 1 우, Sacheon-si, Chukdong-myeon, Baechun-ri, 13 Sep 2001, Paik YH; $1 \sigma^{\nearrow}$ (light trap), Sacheon-si, Waryong-dong, Mt. Waryongsan, Cheongryongsa 11-12 Aug 1999, Sohn JH; 1 지 1 우, Jinju-si, Yugok-dong, Nabul-ri, 16 Aug 2012, Kim TW, Kim OS; 3 万ٓ 1 우 (light trap), Jinju-si, Jinseong-myeon, Gajin-ri, Mt. Wolyasan, 13-14 Aug 1999, Park JS et al.; 1 우 (light trap), Goseong-gun, Sanri-myeon, Dongsan-ri, Hyangnobong, 17-18 Aug 1998, Park JS; 2 우 (light trap), Goseong-gun, Sangrimyeon, Osan-ri, Mt. Odusan, 19-20 Aug 1998, Park JS; 2 우, Goseong-gun, Hail-myeon, Deokho-ri, Seoksan, 20-21 Aug
1998, Jeon JS, Kim SY; 4 우 (light trap), Goseong-gun, Hailmyeon, Yongtae-ri, Mt. Sanseongsan, 18-19 Aug 1998, Park JS; 2 저 2 우 (light trap), Masan-si, Happo-gu, Jinjeon-myeon, Yeoyang-ri, Osilgol, Mt. Yeohangsan, 11-12 Sep 1999, Park JS; 2 우, Namhae-gun, Bonghwa-ri, Hwacheoncheon, 27-28 Aug 1998, An DH, Sohn JH; 2 주 2 우 (light trap), Hapcheongun, Gaya-myeon, Jukjeon-ri, Mt. Gayasan, 19-20 Aug 1999, Park JS; 1 우 (light trap), Hadong-gun, Geumseo-myeon, Galsa-ri, 6-7 Aug 1997, Jeon JS; 1 우 (light trap), Hapcheongun, Bongsan-myeon, Bonggye-ri, Mt. Mangilsan, 22-23 Aug 1998, An TH; 1 우 (light trap), Jinyang, 26-30 Aug 1992, Anonym; $2 \sigma^{\nearrow}$, Geoje-si, Yeoncho-myeon, Songjeong-ri, 11 Jun 2009, Kim TW; Jeollabuk-do: 1 우 (light trap), Muju-gun, 21-25 Aug 1992, Anonym; 1 우 (light trap), Buan-gun, 

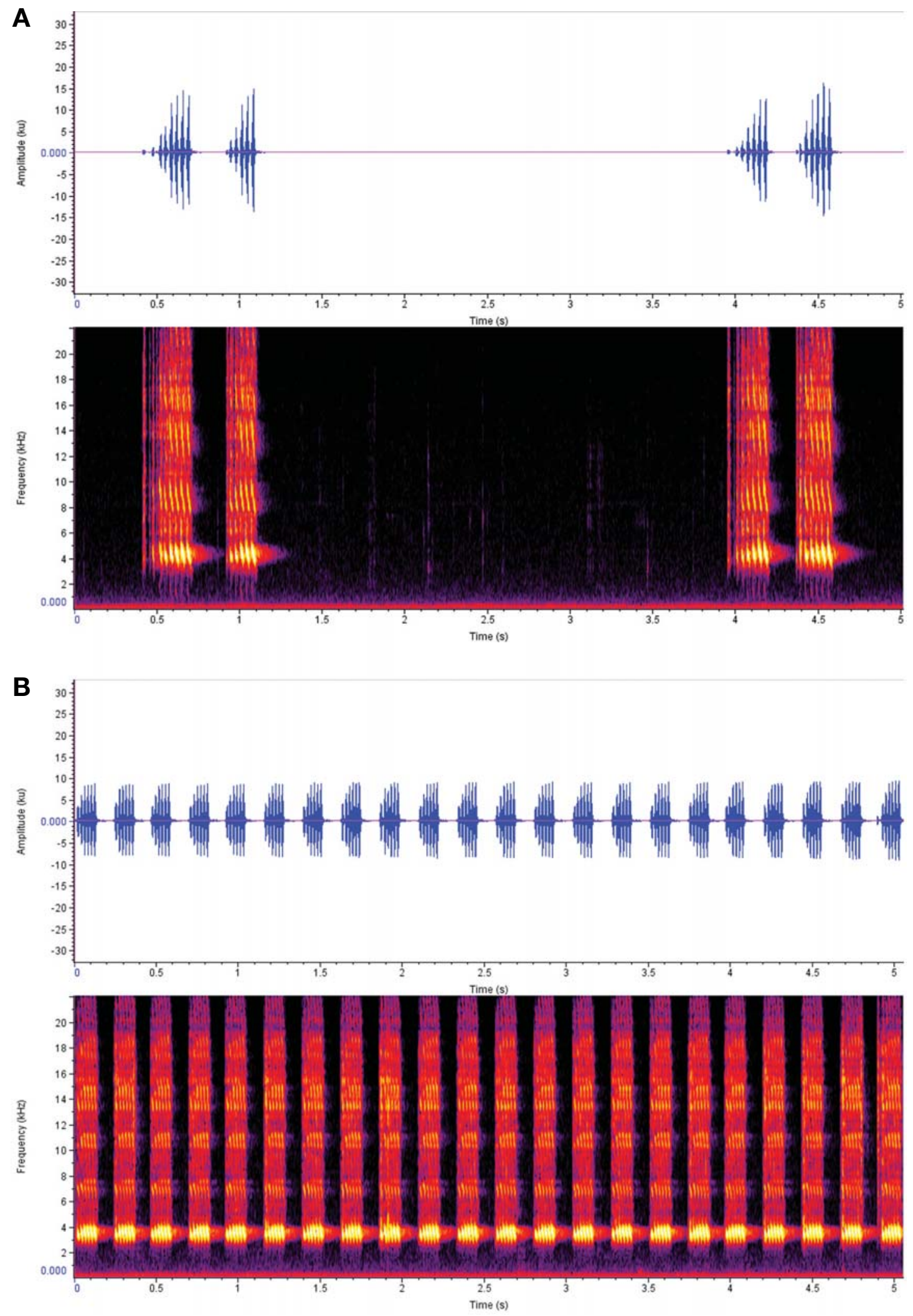

Fig. 5. Calling sounds of Velarifictorus and Lepidogryllus from Korea ( $5 \mathrm{~s}$ at night). A, Velarifictorus aspersus borealis (29 $\left.{ }^{\circ} \mathrm{C}\right)$; $\mathrm{B}$, Velarifictorus micado $\left(27^{\circ} \mathrm{C}\right)$. Continued on next page. 

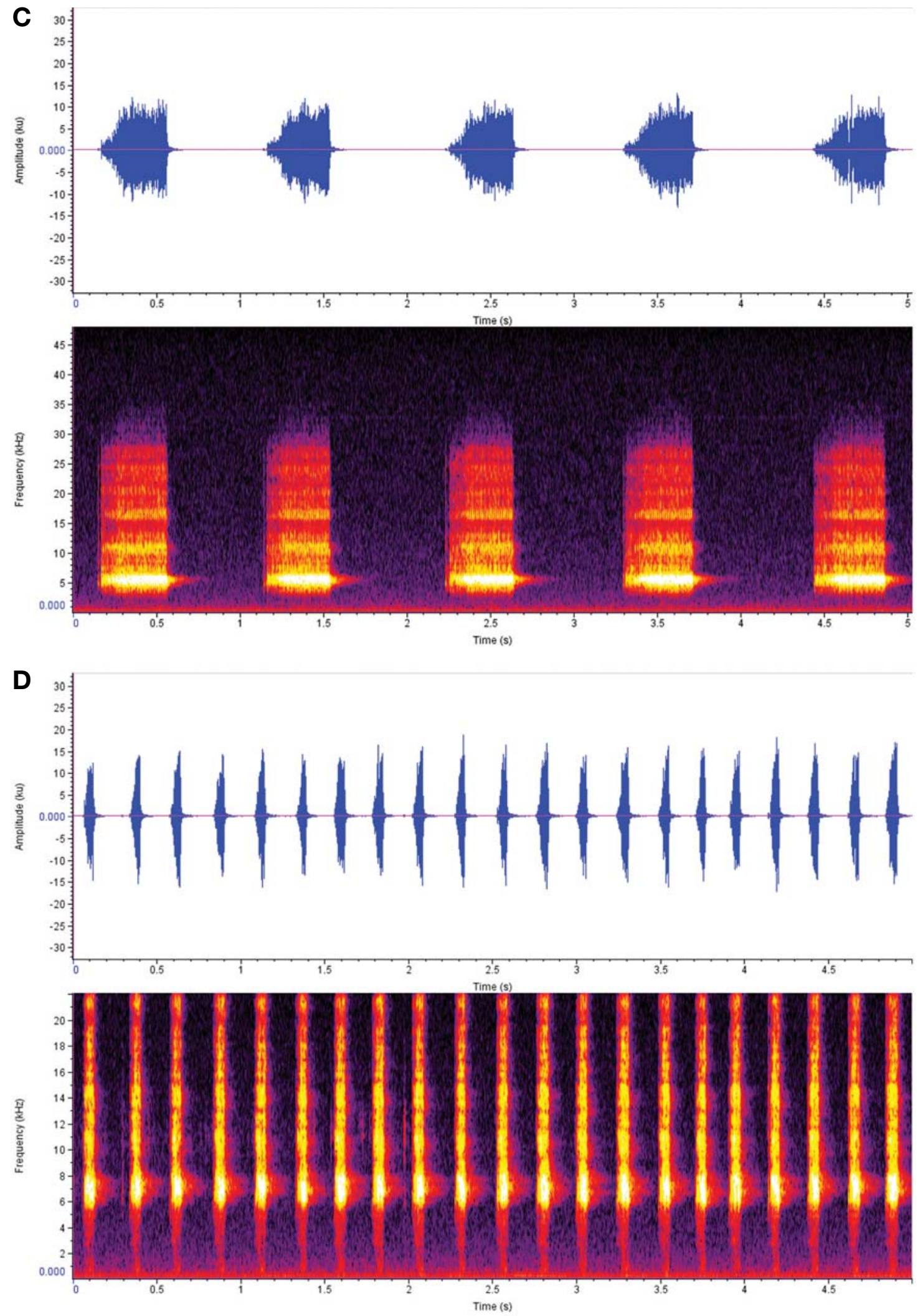

Fig. 5. Continued from previous page. C, Velarifictorus ornatus $\left(28^{\circ} \mathrm{C}\right) ; \mathrm{D}$, Lepidogryllus siamensis $\left(28^{\circ} \mathrm{C}\right)$. 
Byeonsanbando, 25 Jun 1991, Ku HJ; Jeollanam-do: 2 ð, Suncheon-si, Pungdeok-dong, Dongcheon, 3 Sep 2005, Kim TW; 1 우, Gwangyang-si, Mt. Baekwunsan, 12 Aug 1994, Choi HS; 1 우, ibid. 5 Aug 1990, An SB; 1 우, Gwangju-si, Mt. Mudeungsan, 21 May 1998, Oh KO; 1 우, Hampyeonggun, Gigak-ri, 2 Sep 2000, Park WS; 1 우 (light trap), Yeongam-gun, 21-25 Jun 1991, Anonym; 1 우, Mokpo-si, Mt. Yudalsan, 14 Aug 1992, Oh SH; 1 ㅈ3우 (light trap), Yeonggwang-gun, Isl. Anmado, 19 Aug 1989, Kim JI, Lee OJ; $5 \diamond^{\nearrow}$, Wando-gun, Wando-eup, 1 Jul 2010, Kim TW; $2 \sigma^{\nearrow, ~ i b i d . ~} 15$ Jun 2011, Sung KS; Jeju-do: $2 \sigma^{\top}$, Seoguipo-si, Dongheungdong, Jeongbang-pokpo, 9 Jul 2002, Kim TW; 1 우, Namjejugun, Andeok-myeon, Gamsan-ri, Andeokgyegok, 13 Aug 2004, Kim JK, Kim TW.

Description. Male (Fig. 1G): Head shining black; frons vertical; occiput with six longitudinal pale strips posteriorly; a bright strip transverse between lateral ocelli; interocular distance three times wider than horizontal diameter of eye in dorsal view; narrowest distance between antennal sockets 1.5 times wider than horizontal diameter of antennal socket; median ocellus transversely elliptical, located in dorsum of fastigium of vertex with a pale longitudinal line linked below to clypeal suture; clypeal suture transverse three fifths of head, weakly upraised in mid; scape as long as wide; palpi bright. Pronotum as long as head, two times wider than long in dorsal view; anterior and posterior margins pilose, truncated; dorsal surface brownish, mottled; lateral lobe of pronotum a little deeper than pronotal length, anterior lower margin more bright. Tegmina (Fig. 3D) as wide as hind margin of pronotum, hardly reaching abdominal end; harp veins two or three; chordal veins three; mirror area divided, 1.2 times longer than wide; apical area well netted, 1.2 times longer than mirror area; lateral field a little deeper than lateral lobe of pronotum, with 5-6 longitudinal veins. Fore tibia with largely oval-shaped outer tympanum, its length three times longer than wide; inner tympanum small and rounded, 1.2 times longer than wide. Hind femur 2.7 times longer than middle femur. Hind tibia with 5-6 pairs of large dorsal spines on both sides, the largest inner apical spur as long as a half of basitarsus. Hind basitarsus 1.7 times longer than remaining two tarsomeres combined length; 5-7 dorsal spinules on both sides; inner apical spur as long as a half of last tarsomere. Cercus as long as two thirds of hind femur. Subgenital plate shortly conical, its hind margin truncated, deeply notched in mid.

Genitalia (Fig. 4D): Epiphallus with narrow central bridge, its width wider than the length of lateral lobes; median lobe absent; inner and hind margin with a lot of hairs. Ectoparamere slightly surpassing lateral lobe of epiphallus, divided into two triangular projections at apex, which are almost sub- equal in width, strongly and shortly incurved forming forked terminal.

Calling sound (Fig. 5D): Short and quick song phrase; buzzy chirps regularly repeated 4-5 times for one second; a chirp with undulating $11-12$ syllables at $28^{\circ} \mathrm{C}$ condition; dominant frequency spectrum $5.8-7.3 \mathrm{KHz}$.

Female (Fig. 1H): Head with more round frons, mouth parts shorter than in male; interocular distance four times wider than horizontal diameter of an eye. Cercus slightly shorter than ovipositor. Subegenital plate roundly triangular, its hind margin weakly notched. Ovipositor straight needle-shaped, as long as hind femur; apex sharply pointed, subapically dilated, without serration.

Distribution. Korea, Japan, Taiwan, Thailand, India (Oriental Region); Hawaii (introduced). China possibly included in this wide distributional ranges of this cricket, pursuing after records of Modicogryllus confirmatus. Ingrisch (1998) reviewed that Modicogryllus confirmatus is only restricted in Sri Lanka (endemic), outside records of that country are actually misidentifications of Modicogryllus siamensis.

Remarks. This cricket overwinters as nymphal stages in southern localities. Calling sounds of adult can be heard from May. Their sounds mixed with the ones of Velarifictorus ornatus in harmony, but seasonally lasting more continuously than Velarifictorus ornatus to late August due to its bivoltinism (Table 1).

\section{Key to the Species of Velarifictorus and Lepidogryllus from Korea}

1. Fore tibia with large outer and small inner tympana. Male tegmina narrower and elongate, its apical area distinctly longer than mirror area; female tegmina as long male tegmina ............................ Lepidogryllus siamensis

- Fore tibia with only outer tympanum. Male tegmina wider and relatively short, its apical area shorter than or as long as mirror area; female tegmina distinctly shorter than male

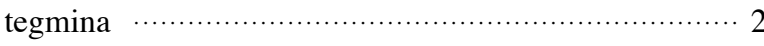

2. Male with elongate mandibles, longer than upper half of head, thus clypeal suture transverse upper than mid in frontal view; ovipositor shorter than hind femur

\section{Velarifictorus aspersus borealis}

- Male with moderate or normal mandibles, shorter than upper half of head, thus clypeal suture transverse lower than mid in frontal view; ovipositor longer than hind femur

3. Head with bright $\wedge$-shaped strip running transversely between lateral ocelli …............... Velarifictorus micado

- Head without distinct transverse strip between lateral ocelli, that reduced to ecdysial feeble line

Velarifictorus ornatus 


\section{ACKNOWLEDGMENTS}

The author would like to express gratitude to Mr. Ichikawa Akihiko(Orthopterological Society of Japan), Dr. S. Ingrisch (Zoological Research Museum Alexander Koenig, Germany), and Dr. A.V. Gorochov (Zoological Institute, Russian Academy of Sciences, St. Petersburg), who provided important papers; Drs. Kim Seung-Tae and Jeong Myeong-Pyo (Seoul National University, Korea), who donated a lot of cricket specimens; Mr. Kim Oh-Sung willingly accompanied my recent field collecting trips, and provided valuable specimens. This work was supported by a grant from the National Institute of Biological Resources (NIBR), funded by the Ministry of Environment (MOE), of the Republic of Korea (NIBR No. 2013-01-032).

\section{REFERENCES}

Alexander RD, Walker TJ, 1962. Two introduced field crickets new to Eastern United States (Orthoptera: Gryllidae). Annals of the Entomological Society of America, 55:90-94.

Bae JD, Lee CE, 1990a. Morphological comparison of stridulatory apparatus of Korean cricket (Gryllidae, Orthoptera). Nature and Life, 20:55-61.

Bae JD, Lee CE, 1990b. Comparative analysis of stridulatory file teeth of Korean cricket (Gryllidae, Orthoptera). Nature and Life, 20:63-68.

Cho PS, 1959. A manual of the Orthoptera of Korea. Human and Natural Science, Korea University, 4:131-198.

Cho PS, 1969. Orthoptera. In: Illustrated encyclopedia of fauna and flora of Korea. Vol. 10 (Ed., Ministry of Education). Samhwa Publishing Co., Seoul, pp. 713-800.

Choo JK, Choi EH, 1983. Studies on the geographic adaptation of field cricket. Korean Journal of Entomology, 13:47-53.

Chopard L, 1928. Revision of the Indian Gryllidae. Records of the Indian Museum, 30:1-36.

Chopard L, 1961. Les divisions du genre Gryllus basees sur l'etude de l'appareil copulateur (Orthoptera, Gryllidae). EOS, Madrid, 37:267-287.

Chopard L, 1967. Gryllides. Pars 12. In: Orthopterorum catalogus (Ed., Beier M). W. Junk's, Gravenhage, pp. 213-500.

Doi H, 1932. Miscellaneous note on insects. Journal of Chosen Natural History Society, 13:30-49.

Eades DC, Otte D, Cigliano MM, Braun H, 2013. Orthoptera Species File Online. Version 2.0/4.0 [Internet]. OSF Online, Accessed 22 Jan 2013, <http://Orthoptera.SpeciesFile.org>.

Gorochov AV, 1978. Crickets of the genera Tartarogryllus and Modicogryllus (Orthoptera, Gryllidae) in Soviet central Asia. Entomological Review, 57:64-69.

Gorochov AV, 1985. On the fauna of Grylloidea(Orthoptera) of China. Entomologicheskoe Obozrenie, 64:89-109.

Gorochov AV, 1986. New and little known crickets (Orthoptera,
Grylloidea) from Middle Asia and adjacent territories. Trudy Zoologicheskogo Instituta Akademii Nauk SSSR, 140:3-15.

Gorochov AV, 1991. Material on the fauna of Gryllinae (Orthoptera, Gryllidae) of Vietnam. Part 1. Trudy Zoologicheskogo Instituta, 240:3-19.

Gorochov AV, 1993. Grylloidea (Orthoptera) of Saudi Arabia and adjacent countries. Fauna of Saudi Arabia, 13:79-97.

Gorochov AV, 1996. New and little known crickets from the collection of the Humboldt University and some other collections (Orthoptera: Grylloidea). Part 1. Zoosystematica Rossica, 4:81-114.

Gorochov AV, 2001. Remarkable examples of convergence and new taxa of Gryllini (Orthoptera: Gryllidae). Zoosystematica Rossica, 9:316-350.

Hua LZ, 2000. List of Chinese insect. Zhongshan University Press, Guangzhou, pp. 32-57.

Ichikawa A, 2001. New species of Japanese crickets (Orthoptera: Grylloidea) with notes on certain taxa. Tettigonia, 3:45-58.

Ichikawa A, Murai T, Honda E, 2000. Monograph of Japanese crickets (Orthoptera: Grylloidea). Bulletin of the Hoshizaki Green Foundation, 4:257-332.

Ingrisch S, 1998. The genera Velarifictorus, Modicogryllus and Mitius in Thailand (Ensifera: Gryllidae, Gryllinae). Entomologica Scandinavica, 29:315-359.

Jin XB, 1994. Chinese cricket culture: an introduction to cultural entomology in China. Cultural Entomology Digest, 3:9-16, 26.

Ju DR, 1993. A series book on the Mt. Baekdusan (animal). Gwahakwon Publishing, Pyeongyang, pp. 1-391.

Ju DR, 1969. Check list of insect classification. Gwahakwon Publishing, Pyeongyang, pp. 16-19.

Kim TW, 2011. A sound guide to Korean crickets (Orthoptera: Ensifera: Grylloidea). National Institute of Biological Resources, Incheon, pp. 1-164.

Kim TW, Puskás G, 2012. Check-list of North Korean Orthoptera based on the specimens deposited in the Hungarian Natural History Museum. Zootaxa, 3202:1-27.

Kwon YJ, Huh EY, 1994. Order 14. Orthoptera. In: Check-list of insects from Korea (Eds., The Entomological Society of Korea, Korean Society of Applied Entomology). Kon-Kuk University Press, Seoul, pp. 48-53.

Kwon YJ, Lee JH, Suh SJ, An SL, Huh EY, Yeo YS, 1996. Korean species list. The Korean National Council for Conservation of Nature, Seoul, pp. 103-104.

Moon TY, Yoon IB, 1994. The list of Orthoptera deposited in the Korean Entomological Institute, II. Oecanthidae, Gryllidae and Gryllotalpidae (Grylloidea). Entomological Research Bulletin (KEI), 20:105-107.

Otte D, 1994. Orthoptera species file 1: Crickets (Grylloidea). The Orthopterists' Society at the Academy of Natural Sciences of Philadelphia, Philadelphia, PA, pp. 1-120.

Otte D, Alexander RD, 1983. The Australian crickets (Orthoptera: Gryllidae). Academy of Natural Sciences of Philadelphia, Monograph, 22:1-477.

Paek MK, Hwang JM, Jung KS, Kim TW, Kim MC, Lee YJ, 
Cho YB, Park SW, Lee HS, Ku DS, Jeong JC, Kim KG, Choi DS, Shin EH, Hwang JH, Lee JS, Kim SS, Bae YS, 2010. Checklist of Korean insects. Nature \& Ecology, Academic Series 2, Seoul, pp. 1-598.

Paik JC, Jung SH, Byun BK, Lee BW, 2010. Forest-inhabiting Orthopteran insects in Korea (Orthoptera). Korean National Arboretum, Sumeungil Co., Seoul, pp. 1-176.

Randell RL, 1964. The male genitalia in Gryllinae (Orthoptera: Gryllidae) and a tribial revision. Canadian Entomologist, 96: 1565-1607.

Saussure H, 1877. Mélanges Orthoptérologiques, Vme Fascicule, Gryllides (I). Mémoires de la Sociétés de Physique et d'Histoire Naturelle de Genève, 25:1-352.

Shiraki T, 1911. Monographie der Grylliden von Formosa, mit der Uebersicht der Japanischen Arten. General gouvernment von Formosa, Taihoku, pp. 1-129.

Shiraki T, 1930. Orthoptera of the Japanese Empire, Part I. Gryllotalpidae and Gryllidae. Insecta Matsumurana, 4:181-252.

Song KD, 1988. Effect of photoperiodism on development of eggs in the three species of field crickets in Korea. Kosin University Theses Collection, 16:355-361.

Song KD, 1989. Geographic variation of the head width in the three species of field crickets in Korea. Kosin University Theses Collection, 17:281-287.

Storozhenko SYu, 2004. Long-horned Orthopterans (Orthoptera: Ensifera) of the Asiatic part of Russia. Dalnauka, Vladivostok, pp. 1-280.

Storozhenko SYu, Paik JC, 2007. Orthoptera of Korea. Dalnauka, Vladivostok, pp. 1-231.

Walker F, 1869. Catalogue of the specimens of Dermaptera Saltatoria and supplement to the Blattaria in the collection of the British Museum I-II. British Museum, London, pp. 1-423.

Walker TJ, 1977. Japanese burrowing cricket widely established in southeastern United States. Florida Entomologist, 60: 308-309.

Wu CF, 1935. Catalogus Insectorum Sinensium I. The Fan Memorial Institute of Biology, Peiping, pp. 15-214.

Yin HS, Liu XW, 1995. Synopsis on the classification of Grylloidea and Gryllotalpoidea from China. Shanghai Scientific and Technological Literature Publishing House, Shanghai, pp. 1-237.

Received June 3, 2013

Revised July 18, 2013

Accepted July 25, 2013 